\title{
The usefulness of F-18 FDG PET/CT- mammography for preoperative staging of breast cancer: comparison with conventional PET/CT and MR-mammography
}

\author{
Eun-Ha Moon ${ }^{1,3}$, Seok Tae Lim¹,2, Yeon-Hee Han¹, Young Jin Jeong ${ }^{4}$, Yun-Hee Kang ${ }^{5}$, \\ Hwan-Jeong Jeong ${ }^{1,2}$, Myung-Hee Sohn ${ }^{1,2}$

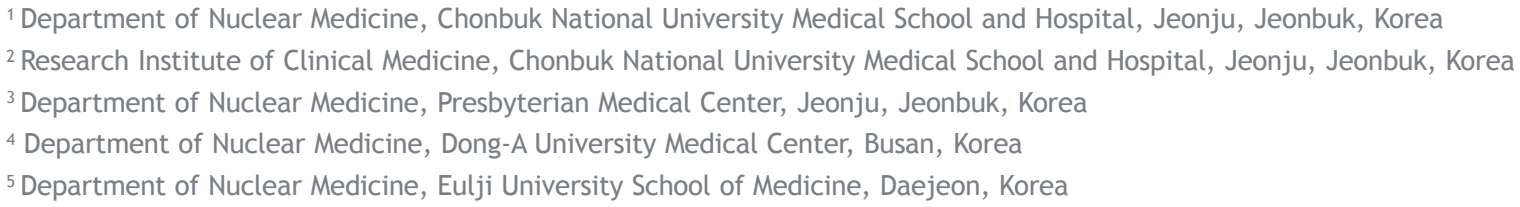

Radiol Oncol 2013; 47(4): 390-397.

Received 20 November 2012

Accepted 14 April 2013

Correspondence to: Seok Tae Lim, Department of Nuclear Medicine, Chonbuk National University Medical School and Hospital, San 2-20 Geumam-Dong, Deokjin-Gu, Jeonju, Jeonbuk, 561-180, South Korea. Phone: +82-63-250-1174; Fax: +82-63-255-1172; E-mail: stlim@jbnu.ac.kr Disclosure: No potential conflicts of interest were disclosed.

Background. The objective of the study was to compare the diagnostic efficacy of an integrated Fluorine-18 fluorodeoxyglucose (F-18 FDG) PET/CT-mammography (mammo-PET/CT) with conventional torso PET/CT (supine-PET/CT) and MR-mammography for initial assessment of breast cancer patients.

Patients and methods. Forty women (52.0 \pm 12.0 years) with breast cancer who underwent supine-PET/CT, mammo-PET/CT, and MR-mammography from April 2009 to August 2009 were enrolled in the study. We compared the size of the tumour, tumour to chest wall distance, tumour to skin distance, volume of axillary fossa, and number of metastatic axillary lymph nodes between supine-PET/CT and mammo-PET/CT. Next, we assessed the difference of focality of primary breast tumour and tumour size in mammo-PET/CT and MR-mammography. Histopathologic findings served as the standard of reference.

Results. In the comparison between supine-PET/CT and mammo-PET/CT, significant differences were found in the tumour size (supine-PET/CT: $1.3 \pm 0.6 \mathrm{~cm}$, mammo-PET/CT: $1.5 \pm 0.6 \mathrm{~cm}, \mathrm{p}<0.001$ ), tumour to thoracic wall distance $(1.8 \pm 0.9 \mathrm{~cm}, 2.2 \pm 2.1 \mathrm{~cm}, \mathrm{p}<0.001)$, and tumour to skin distance $(1.5 \pm 0.8 \mathrm{~cm}, 2.1 \pm 1.4 \mathrm{~cm}, \mathrm{p}<0.001)$. The volume of axillary fossa was significantly wider in mammo-PET/CT than supine-PET/CT $\left(21.7 \pm 8.7 \mathrm{~cm}^{3} \mathrm{vs} .23 .4 \pm 10.4 \mathrm{~cm}^{3}\right.$, $\mathrm{p}=0.03)$. Mammo-PET/CT provided more correct definition of the T-stage of the primary tumour than did supine-PET/ CT (72.5\% vs. 67.5\%). No significant difference was found in the number of metastatic axillary lymph nodes. Compared with MR-mammography, mammo-PET/CT provided more correct classification of the focality of lesion than did MR-mammography (95\% vs. $90 \%$ ). In the T-stage, $72.5 \%$ of cases with mammo-PET/CT and $70 \%$ of cases with MRmammography showed correspondence with pathologic results.

Conclusions. Mammo-PET/CT provided more correct definition of the T-stage and evaluation of axillary fossa may also be delineated more clearly than with supine-PET/CT. The initial assessment of mammo-PET/CT would be more useful than MR-mammography because the mammo-PET/CT indicates similar accuracy with MR-mammography for decision of T-stage of primary breast tumour and more correct than MR-mammography for defining focality of lesion.

Key words: breast cancer; fluorodeoxyglucose; positron emission tomography; MRl; mammography 


\section{Introduction}

Breast cancer is the most common cancer and the second leading cause of cancer-related death in women in western countries. ${ }^{1}$ Currently, breast cancer is diagnosed by triple assessment of physical examination, radiologic evaluation, and histopathologic confirmation. In particular, radiologic evaluation is more important when the size of tumour is too small. ${ }^{2,3}$ Treatment strategy of breast cancer is dependent on the size of the breast, size or extent of the tumour, infiltration, and lymph node metastasis, et cetera. Therefore, once breast cancer is diagnosed, accurate staging of the tumour is extremely important as it influences appropriate treatment decision and determines prognosis. ${ }^{4}$

Initial breast cancer staging has been based on a multimodality approach: X-ray mammography, ultrasonography, MRI, sentinel lymph node biopsy, and bone scintigraphy. ${ }^{5}$ X-ray mammography is the most widely used technique for evaluation of the primary tumour in both symptomatic and asymptomatic patients. ${ }^{6-8}$ However, because of dense breasts, X-ray mammography is less sensitive for Korean women, and underestimates the size or focality of the primary tumour. ${ }^{23,9,10}$ Correlation of $\mathrm{X}$-ray mammography findings with breast ultrasonography and MRI has been helpful for differential diagnosis of a primary breast lesion and for detection of occult breast tumours. ${ }^{11-13}$ However, this multimodality approach is invasive, as well as time and cost consuming. Thus, a noninvasive, single-session approach for breast cancer staging may be desirable. ${ }^{14-16}$

Fluorine-18 fluorodeoxyglucose positron emission tomography/computed tomography (F-18 FDG PET/CT) allows for accurate staging of various types of malignancies and for acquisition of whole-body imaging. ${ }^{17,18}$ Thus, PET/CT has high sensitivity in detection of primary tumour and axillary lymph node metastasis, as well as distant metastasis, in patients with breast cancer. In addition, PET/CT is beneficial for use in monitoring of therapy. However, with CT as part of PET/CT, tumour delineation is more difficult to determine than with MRI up to now, PET/CT has been used predominantly for evaluation of distant metastases. ${ }^{19-23} \mathrm{MR}-$ mammography, as well as X-ray mammography, in conjuction with ultrasonography, has remained the method of choice for imaging of the primary tumour of the breast. Patient positioning, which is similar to that used in MR-mammography (PET/ CT-mammography), may provide more accurate information on the primary tumour and axillary lymph node in PET/CT. However, the diagnostic accuracy of this PET/CT-mammography algorithm has not yet been defined.

The purpose of this study was to assess the usefulness and feasibility of PET/CT-mammography for preoperative staging of breast cancer and to compare the capability of measurement and delineation of lesion of PET/CT-mammography to those of conventional torso F-18 FDG PET/CT and MRmammography.

\section{Patients and methods}

\section{Patients}

Forty women (mean age; $52.0 \pm 12.0$ years) with breast cancer who underwent conventional torso F-18 FDG PET/CT (supine-PET/CT), PET/ CT-mammography (mammo-PET/CT) (PET/CTmammography was acquired after the conventional torso PET/CT), and MR-mammography from April 2009 to August 2009 were enrolled (mean interval between MR-mammography and PET/CTmammography: $1.7 \pm 3.9$ days, range: 0-23 days). All patients were confirmed breast cancer by biopsy before the diagnostic imagings were acquired. We compared the primary tumour size (longest diameter of lesion in trans-axial image), tumour to thoracic wall distance ( $\mathrm{T}-\mathrm{C}$ distance, maximal distance from tumour to thoracic wall in trans-axial image), tumour to skin distance (T-S distance, maximal distance from tumour to skin in trans-axial image), volume of ipsilateral axillary fossa, and number of suspected metastatic axillary lymph nodes between supine-PET/CT and mammo-PET/ $\mathrm{CT}$, retrospectively. Next, we evaluated the difference of the focality of the primary tumour and the size of the tumour in mammo-PET/CT and MRmammography, retrospectively. Histopathologic findings served as the standard of reference. The protocol was approved by the Institutional Review Board of Chonbuk National University Hospital.

\section{Imaging protocol Supine-PET/CT and mammo-PET/CT}

Whole body F-18 FDG PET/CT scans were obtained on a BiographTruepoint 40 PET/CT (Siemens, Berlin, Germany). Patients fasted for at least 6 hours before receiving an intravenous injection of F-18 FDG (mean: $429.0 \pm 59.0 \mathrm{MBq}$ $(11.6 \pm 1.6 \mathrm{mCi})$, range: $259.0-592.0 \mathrm{MBq}(7-16$ $\mathrm{mCi})$ ). Prior to injection of F-18 FDG, blood glucose levels were checked in order to determine 


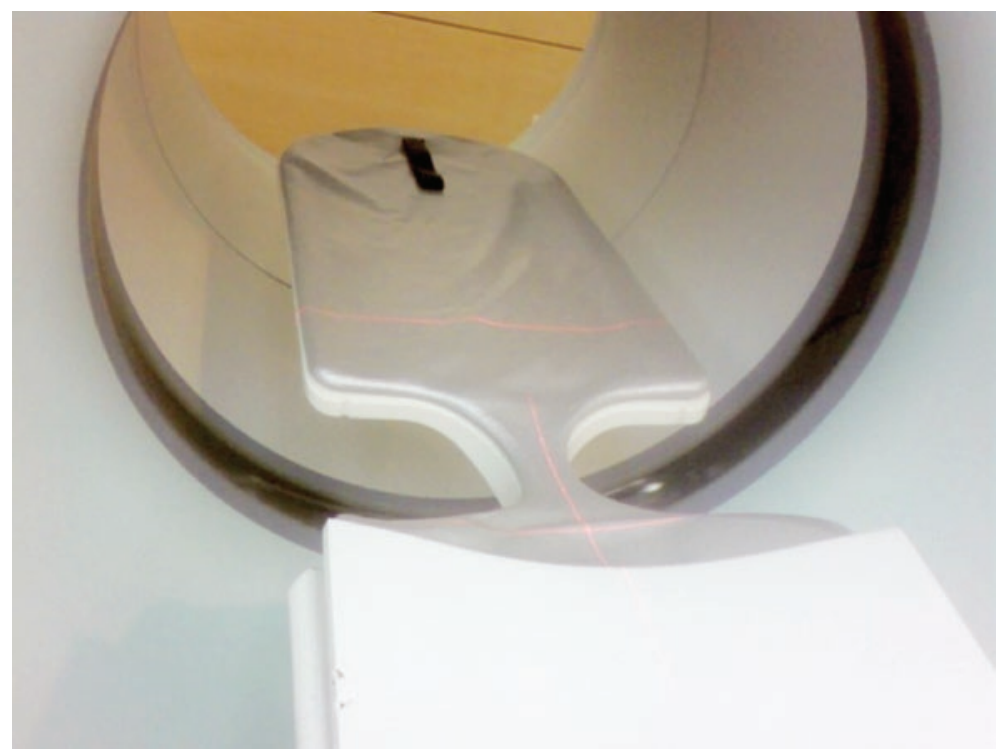

FIGURE 1. Breast positioning device. The device is constructed for prone breast positioning. sequence $(3,200 / 96$ [repetition time ms/echo time ms]). A T1-weighted 3-dimensional, fat-suppressed fast spoiled gradient-echo sequence (11.0/4.8; flip angle, $90^{\circ}$; bandwidth, $150 \mathrm{~Hz}$ ) was then performed before and 4 times sequentially after a rapid bolus intravenous injection of $0.1 \mathrm{mmol} / \mathrm{kg}$ body weight of gadolinium at an injection rate of $2.0 \mathrm{ml} / \mathrm{s}$. Image acquisition began immediately after administration of the contrast material and saline bolus.

\section{Image analyses}

Analysis of PET/CT images was performed by two nuclear medicine physicians and MRmammography was read by two radiologists. The evaluating physicians were blinded to the results of the other imaging procedures.

\section{Supine-PET/CT and mammo-PET/CT}

Assessments of the primary tumour, axillary lymph node metastasis, and distant metastasis with PET/CT were based on qualitative and quantitative assessments. PET/CT data were assessed qualitatively for regions of focally increased glucose metabolism and semi-quantitatively by maximal standardized uptake values (SUVmax). A lesion was determined as malignancy or metastasis on PET/CT if FDG uptake was higher than that in the surrounding tissue on qualitative analysis; the SUVmax was checked for the corresponding lesions. We also calculated the SUVmax ratio of primary breast lesion (SUVmax of primary lesion/ SUVmax of adjacent muscle) and suspected metastatic lymph node in the axillary fossa (SUVmax of ipsilateral, metastatic axillary lymph node/ SUVmax of adjacent muscle).

A breast lesion was suspected as malignant if it showed contrast enhancement, compared with the surrounding tissue (attenuation measurement with regions of interest (ROI) and expressed in Hounsfield unit (HU)) and elevated FDG uptake, compared with the adjacent breast tissue. The volume of ipsilateral axillary fossa $\left(\mathrm{cm}^{3}\right)$ was evaluated for potential lymph node metastasis. Axillary fossa was assessed by measuring the area of axillary fat (between the outer margin of the latissimusdorsi/major teres muscle and minor/major pectoralis muscle). ${ }^{24}$ Lymph nodes were graded as malignant or benign based on their size and morphologic pattern (cross-sectional short axis diameter of more than $10 \mathrm{~mm}$, loss of fatty hilum or cortical thickening supported the diagnosis of lymph node metastasis). In addition, FDG uptake of axillary lymph node was assessed using qualitative and quantitative analyses. 
TABLE 1. Comparison of conventional whole body PET/CT and PET/CT-mammography in characteristics of primary breast lesions and ipsilateral axillary area

\begin{tabular}{lccc} 
& Whole Body PET/CT $(\mathrm{n}=\mathbf{4 0})$ & PET/CT-mammography $(\mathrm{n}=\mathbf{4 0})$ & $\mathrm{p}$ value \\
\hline Tumour size $(\mathrm{cm})$ & $1.3 \pm 0.6$ & $1.5 \pm 0.6$ & $<0.001$ \\
Mean T-C $(\mathrm{cm})$ & $1.8 \pm 0.9$ & $2.2 \pm 2.1$ & $<0.001$ \\
Mean T-S $(\mathrm{cm})$ & $1.5 \pm 0.8$ & $2.1 \pm 1.4$ & $<0.001$ \\
Axillary area $\left(\mathrm{cm}^{3}\right)$ & $21.7 \pm 8.7$ & $23.4 \pm 10.4$ & 0.03 \\
Compatibility with pathologic T-staging (\%) & $67.5(27 / 40)$ & $72.5(29 / 40)$ & 0.63 \\
\hline
\end{tabular}

* Tumour-Chest wall distance; † Tumour-Skin distance; $n=$ number of patients

Supine-PET/CT and mammo-PET/CT were compared for assessment of the primary tumour and axillary lymph nodes. All PET/CT images were reviewed in 3 orthogonal planes (axial, coronal, and sagittal).

\section{MR-mammography}

Determination of malignancy on MR-mammography was based on assessment of tumour morphological characteristics, as well as the pattern of contrast-enhancement. Breast lesions were rated according to the American College of Radiology Breast Imaging Reporting and Data System lexicon. Both the morphologic appearance (size, shape, and enhancement pattern) and the temporal enhancement pattern were evaluated. Time-signal-intensity curves (progressive, plateau, or washout) were generated for all enhancing lesions.

Evaluation and staging of malignant lesions was performed according to the American Joint Committee on Cancer (AJCC) staging classification. The 2 imaging modalities (PET/CT and MRmammography) were compared for $\mathrm{T}$ stage and focality of the primary tumour. Findings of focality of the primary tumour with mammo-PET/CT and MR-mammography were classified as unifocal (a single lesion in 1 quadrant), multifocal (more than 2 lesions in the same quadrant), or multicentric lesions (more than 2 quadrants affected by breast cancer, or distance between breast cancer lesions more than $4 \mathrm{~cm}$ within 1 quadrant).

\section{Data analyses}

Accuracy for delineation of primary tumour and axillary lymph node metastasis among the supine$\mathrm{PET} / \mathrm{CT}$, mammo-PET/CT, and MR-mammography was calculated. $\mathrm{T}$ stage of the primary tumour was assessed with supine-PET/CT, mammo-PET/CT, and MR-mammography. Axillary lymph node was assessed between the supine-PET/CT and mammoPET/CT. In addition, the ability for accurate differentiation of unifocal, multifocal, and multicentric lesions was compared between mammo-PET/CT and MR -mammography. The final diagnosis was served by the histopathologic results.

\section{Statistical Analyses}

Accuracy for assessment of the primary tumour and axillary lymph nodes was calculated according to a patient-based analysis. Differences between images and histopathologic results were evaluated using a paired t-test, Pearson chi-square test, and Fisher's exact test. A $p$-value of less than 0.05 indicated a significant difference. SPSS version 12.0 (IBM, New York, USA) was used in performance of all statistical analyses.
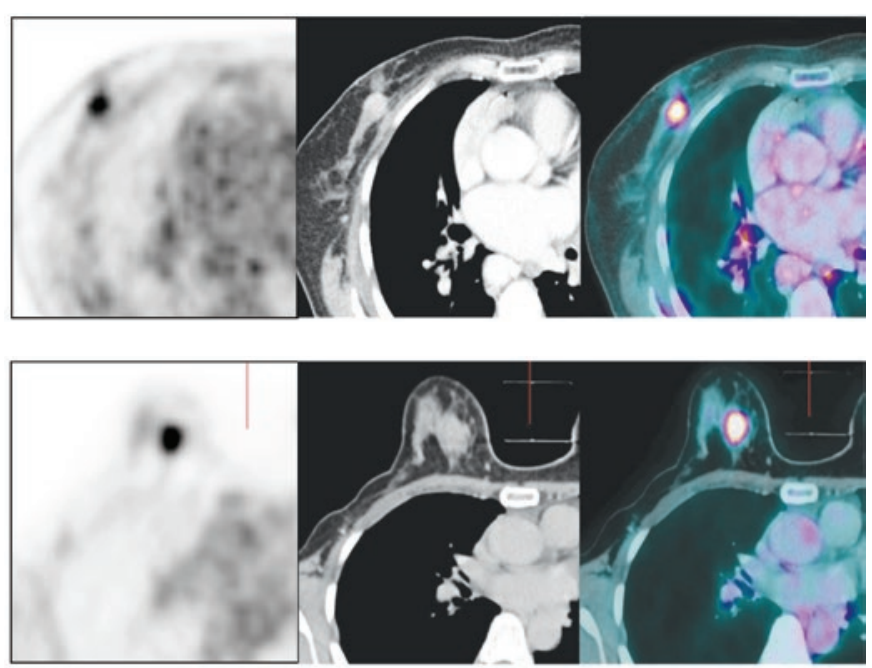

FIGURE 2. A woman with right breast cancer performed the supine-PET/CT and mammo-PET/CT. A The primary tumour was abutted on the chest wall in the supinePET/CT. BThe tumour could be more clearly distinguished from the chest wall in the mammo-PET/CT. 
TABLE 2. T-stage of resected breast cancer and number of patients correctly staged with PET/CT-mammography and MR-mammography

\begin{tabular}{lccc}
\hline T-stage & Pathologic Confirmation $(\mathrm{n}=40)$ & $\begin{array}{c}\text { Staged with } \\
\text { PET/CT-mammography }(\mathrm{n}=40)\end{array}$ & $\begin{array}{c}\text { Staged with MR-mammography } \\
(\mathrm{n}=\mathbf{4 0})\end{array}$ \\
\hline Tla & 6 & $2(33.3 \%)$ & - \\
Tlb & 1 & $1(100 \%)$ & $1(100 \%)$ \\
Tlc & 21 & $18(87.5 \%)$ & $8(90.5 \%)$ \\
T2 & 11 & - & $8(72.7 \%)$ \\
T3 & 1 & - & - \\
T4 & 0 & $29(72.5 \%)$ & $28(70 \%)$ \\
Total & 40 & & - \\
\hline
\end{tabular}

\section{Results}

\section{Assessment of the primary tumour}

Pathologic biopsy showed that invasive ductal carcinoma, invasive lobular carcinoma and invasive micropapillary carcinoma were found $77.5 \%$ (31/40), 5\% (2/40) and 5\% (2/40) of patients. Ductal carcinoma in situ (DCIS) and the others (small cell carcinoma and tubular carcinoma) were found $7.5 \%(3 / 40)$ and $5 \%(2 / 40)$ of patients.

\section{Comparison between supine-PET/CT and mammo-PET/CT}

The mean size of primary tumour lesions, in the longest axis dimension, was significantly larger on mammo-PET/CT than on supine-PET/CT (supinePET/CT: $1.3 \pm 0.6 \mathrm{~cm}$, mammo-PET/CT: $1.5 \pm 0.6$ $\mathrm{cm}, \mathrm{p}<0.001)$. The difference of tumour size between PET/CT imaging and histopathologic result was $0.5 \pm 1.1 \mathrm{~cm}$ on supine-PET/CT and $0.2 \pm 1.2$ $\mathrm{cm}$ on mammo-PET/CT. Thus, the real size of the primary tumour was more accurate on mammo$\mathrm{PET} / \mathrm{CT}$ than on supine-PET/CT. The mean tumour to chest wall distance (supine-PET/CT: $1.8 \pm$ $0.9 \mathrm{~cm}$, mammo-PET/CT: $2.2 \pm 2.1 \mathrm{~cm}, \mathrm{p}<0.001)$ and tumour to skin distance (supine-PET/CT: 1.5 $\pm 0.8 \mathrm{~cm}$, mammo-PET/CT: $2.1 \pm 1.4 \mathrm{~cm}, \mathrm{p}<0.001)$ were significantly longer on mammo-PET/CT than on supine-PET/CT, indicating better delineation of the tumour from the chest wall and skin (Figure 2, Table 1). The mean SUVmax of the primary tumour was $7.6 \pm 5.8$ (range: 1.4-20.8) on supine-PET/CT and $8.0 \pm 6.2$ (range: $1.8-21.4$ ) on mammo-PET/CT $(p=0.02)$.The mean SUVmax ratio of the primary tumour showed 5.4 (range: 1.4 30.8) in the supinePET/CT and 5.7 (1.3 23.4) in the mammo-PET/CT. No significant difference was showed between the supine-PET/CT and mammo-PET/CT statistically $(\mathrm{p}=0.18)$.
Six patients were proven with T1a stage, and 1 patient with T1b, 21 patients with T1c, 11 patients with T2, and 1 patient with T3 by histopathologic confirmation. None of the patients had T4 stage of breast cancer. Characterization of $\mathrm{T}$ stage in breast cancer lesions was corrected in $27(67.5 \%)$ of 40 patients on supine-PET/CT and 29 (72.5\%) on mammo-PET/CT, compared with histopathologic confirmation (Table 1 ). No statistically significant difference was observed $(\mathrm{p}=0.63)$.

\section{Comparison between mammo-PET/CT and MR- mammography}

In comparison of $\mathrm{T}$ stage, mammo-PET/CT provided correct classification of $\mathrm{T}$ stage of the primary tumour in $72.5 \%$ of cases. In patients with T1a, T1b, and T1c stage, mammo-PET/CT correctly found 2 $(33.3 \%)$ of 6 patients, $1(100 \%)$ of 1 patient, and 18 (87.5\%) of 21 patients, respectively. Mammo-PET/ CT also correctly found 8 (72.7\%) of 11 patients in the T2 stage and none of the patients had T3 or T4 stage on mammo-PET/CT. MR-mammography provided correct characterization of $\mathrm{T}$ stage in a total of $28(70 \%)$ of 40 patients. In patients with T1 (T1a-T1c) stage, MR-mammography found $1(100 \%)$ of 1 patient with T1b, and $19(90.5 \%)$ of 21 patients with T1c. In T2 stage patients, MRmammography correctly found $8(72.5 \%)$ of 11 patients and none of the patients had T3 or T4 stage in MR-mammography $(p=0.81)$ (Table 2). In measuring the size of primary tumour, one case showed more correct result in the mammo-PET/CT than the MR-mammography although the $\mathrm{T}$ stage was not changed.

In the focality of primary breast lesions, mammo-PET/CT provided correct characterization of $38(95 \%)$ of 40 patients and MR-mammography found 36 (90\%) of 40 patients. Solitary, multifocal, and multicentric lesions were found in 33,2 , and 
TABLE 3. Comparison of PET/CT-mammography and MR-mammography in focality of primary breast lesions

\begin{tabular}{lccc}
\hline Focality & Pathologic Confirmation $(n=40)$ & PET/CT-mammography $(n=40)$ & MR-mammography $(n=40)$ \\
\hline Solitary & 33 & 32 & 31 \\
Multifocal & 2 & 1 & 1 \\
Multicentric & 5 & 5 & 4 \\
Total & & $38(95 \%)$ & $36(90 \%)$ \\
\hline
\end{tabular}

5 patients in histopathologic confirmation. Finally, mammo-PET/CT found 32 of 33 patients with a solitary lesion, 1 of 2 patients with multifocal lesions, and 5 of 5 patients with multicentric lesions. MRmammography correctly found 31 of 33 patients with a solitary lesion, 1 of 2 patients with multifocal lesions, and 4 of 5 patients with multicentric lesions $(p=0.68)$ (Table 3).

\section{Axillary lymph node and distant metastasis}

In axillary lymph node dissection, intra-axillary lymph node metastasis was histopathologically proven in 18 of 40 patients. PET/CT provided correct detection of intra-axillary metastastic lymph nodes in 14 of 18 patients and the other 4 patients showed no significant FDG uptake and negative imaging findings. No significant differences between supine-PET/CT and mammo-PET/CT were evaluated in the detection of the number of metastatic axillary lymph nodes. Sensitivity, specificity, and accuracy of PET/CT (supine-PET/CT and mammo-PET/CT) for detection of metastatic axillary lymph nodes were $77.8 \%, 86.4 \%$, and $82.5 \%$, respectively. The mean SUVmax of these metastatic lymph nodes was $4.8 \pm 3.9$ (range: 1.4 - 13.0) on supine-PET/CT and $5.3 \pm 4.4$ (range: $2.0-14.3$ ). The mean SUVmax ratio of these metastatic lymph nodes showed 1.3 (range: 1.0 - 10.4) in the supinePET/CT and 1.4 (range: 1.2 - 10.6). There was a no significant difference statistically $(\mathrm{p}=0.25)$.

Although no significant differences between supine-PET/CT and mammo-PET/CT were detected in the number of metastatic axillary lymph nodes, the volume of ipsilateral axillary fossa was also significantly wider on mammo-PET/CT than on supine-PET/CT (supine-PET/CT: $21.7 \pm 8.7$ $\mathrm{cm}^{3}$, mammo-PET/CT: $23.4 \pm 10.4 \mathrm{~cm}^{3}, \mathrm{p}=0.03$ ) (Figure 3, Table 1).

No distant metastases were found in any of the patients in this study; therefore, PET/CT including mammo-PET/CT did not influence further therapeutic decisions in any patient.

\section{Discussions}

According to the Central Cancer Registry Program 2009 , the frequency of breast cancer was $15.1 \%$ among women in 2007 and it was reported to be second in cancer occurrence among Korean women, following thyroid cancer. ${ }^{25}$ In Korea, breast cancer has shown a significant increase every year. Therefore, early detection of breast cancer is associated with attainment of cure through early intervention. $^{26}$

Initial breast cancer staging has been based on a multimodality approach: X-ray mammography, ultrasonography, MRI, sentinel lymph node biopsy, and bone scintigraphy. However, this multimodality approach is time consuming and uncomfortable for patients. Thus, a noninvasive, singlesession approach to breast cancer staging may be desirable. ${ }^{11-16}$

F-18 FDG PET/CT has been reported to be useful in staging, restaging, and monitoring of treatment response in cancer patients. PET/CT has high sensitivity in detection of the primary tumour and axillary lymph node metastasis, as well as distant metastasis in patients with breast cancer. However, with CT as part of PET/CT, tumour delineation is more difficult to determine than with MRI and PET/CT has been used predominantly for evaluation of distant metastases. ${ }^{19-23}$

Because the glandular and fatty tissues of the breast are not uncompressed, prone breast positioning like that used in MR-mammography has a substantial advantage in better differentiation of the primary tumour from its adjacent structures and may improve the assessment of potential infiltration of tumour into the chest wall or skin of the breast. Theoretically, prone positioning may provide more accurate information on the primary tumour and axillary lymph node in PET/ CT. In addition, due to enhanced anatomical visualization, the axillary fossa may be evaluated more easily for potential metastatic lymph nodes. MRI 

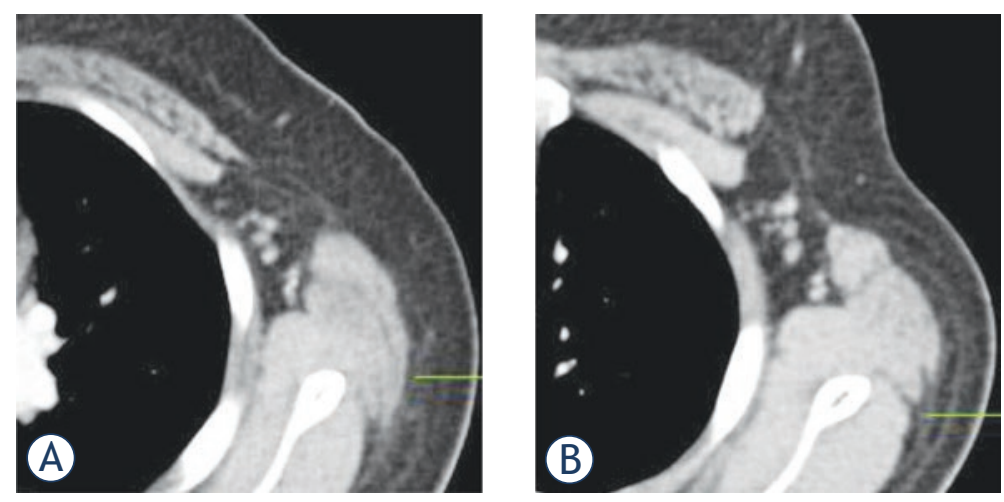

FIGURE 3. Assessment of axillary fossa in the woman with left breast cancer. A The volume of left axillary fossa measured $15 \mathrm{~cm}^{3}$ in the supine-PET/CT. B The volume of axillary fossa measured $24 \mathrm{~cm}^{3}$ in the mammo-PET/CT and the assessment of axillary fossa for the potential of metastatic axillary lymph node can be more clear and easy tudy group.

has been known to be a sensitive but less specific imaging modality for detection and characterization of breast cancer lesions. Findings from the current study suggest that the protocol for PET/ CT-mammography may have similar sensitivity to MR-mammography for detection of intra-mammary lesions. ${ }^{27-29}$ However, the diagnostic accuracy of PET/CT-mammography has not yet been defined. We assessed the diagnostic accuracy of PET/CTmammography as a single diagnostic modality in preoperative staging of breast cancer patients, compared with conventional PET/CT and MRmammography.

Orel et al. ${ }^{30}$ reported that MRI showed high sensitivity $(91-100 \%)$ for staging of invasive breast cancer, and other studies have also reported that the sensitivity of MRI was 93\%; however, specificity was relatively low $(65 \%)$ due to the lack of ability to characterize enhancing lesions as benign or malignant. ${ }^{31-33}$ MRI showed $85 \%$ accuracy in breast cancer stage; Heusner et al. ${ }^{34}$ reported that MRI showed $77 \%$ accuracy for T stage, and $73 \%$ accuracy for focality of the primary tumour.

Antoch et al. ${ }^{12}$ reported that MRI showed 52\% accuracy for $\mathrm{T}$ stage and $79 \%$ accuracy for $\mathrm{N}$ stage; therefore, this result showed lower accuracy than that of another study due to the use of whole body MRI. This study showed that MR-mammography had $70 \%$ accuracy for T stage, and $90 \%$ for focality of the primary tumour. In comparison with previous studies, MR-mammography showed slightly lower accuracy in $\mathrm{T}$ stage and greater accuracy in decision of focality.

In a previous study of FDG PET for staging of breast cancer, Samson et al. ${ }^{35}$ reported that FDG
PET had $89 \%$ sensitivity, and Antoch et al. ${ }^{12}$ reported that FDG PET/CT showed $80 \%$ accuracy in $\mathrm{T}$ stage, and $93 \%$ accuracy in $\mathrm{N}$ stage. According to this data, conventional PET/CT (supine-PET/ $\mathrm{CT}$ ) showed $67.5 \%$ accuracy in $\mathrm{T}$ stage and mammo-PET/CT showed $72.5 \%$ accuracy in T stage, and $95 \%$ accuracy for focality of the primary lesion. Supine-PET/CT and mammo-PET/CT showed similar accuracy $(77.8 \%)$ for $\mathrm{N}$ stage. This study showed slightly lower accuracy than that of current studies in $\mathrm{T}$ and $\mathrm{N}$ stage of breast cancer patients; however, appropriate comparison between the results would be difficult because only a few studies of mammo-PET/CT in breast cancer staging have been conducted. However, like the preceding study, the current study suggests that this PET/CT protocol for breast cancer may be similar to MRI for detection of intra-mammary cancer lesions. The accuracy of assessment of the primary tumour was also similar between mammo-PET/CT and MRmammography in this study.

Heusner et al. reported that the area of axillary fossa $\left(\mathrm{cm}^{2}\right)$ was significantly wider on mammoPET/CT than on supine-PET/CT and anatomical structures of the axilla may be more easily differentiated from one another. ${ }^{24}$ Although we assessed the volume of axillary fossa and no significant differences were detected in the number of metastatic axillary lymph nodes, this data also showed that the volume of axillary fossa was wider on mammoPET/CT than on supine-PET/CT.

None of the patients in this study had distant metastasis; however, Heusner et al. reported that PET/CT influenced further therapeutic decisions in 5 patients by detecting the unexpected distant metastasis or synchronous malignancy. ${ }^{34}$

There are some technical limitations in acquisition of whole body mammo-PET/CT. We acquired the mammo-PET/CT image after conventional supine-PET/CT. A whole body mammo-PET/CT protocol, instead of the combined whole body supine-PET/CT with mammo-PET/CT, should be discussed, as this would reduce the examination time. However, patient tolerance may limit this proto$\mathrm{col}$, because the acquisition time can be up to 90 minutes. Use of state-of-the-art multislice PET/CT systems will further reduce the examination times, compared with PET/CT scanners, with fewer detector rows. In this setting, a whole body mammoPET/CT may be clinically feasible.

Based on our data, mammo-PET/CT improved the accuracy in initial staging of breast cancer and showed that the evaluation of axillary fossa was more clearly and easily, compared with supine- 
PET/CT. Also mammo-PET/CT indicated the similar accuracy with MR-mammography in T stage and was more correct than MR-mammography for defining focality of lesion. Furthermore, PET/CT can acquire whole body image, so has the advantage for detecting the unexpected, distant metastasis and changing the treatment strategy.

\section{Acknowledgement}

This study was supported by a grant from the National R\&D Program for Cancer Control, Ministry Health, Welfare and Family Affairs, Republic of Korea (0620220 and 0720420).

\section{References}

1. Jemal A, Siegel R, Ward E, Murray T, Xu J, Smigal C, et al. Cancer statistics, 2006. CA Cancer J Clin 2006; 56: 106-30.

2. Kopans DB. The positive predictive value of mammography. Am J Roentgenol 1992; 158: 521-6.

3. Bird RE, Wallace TW, Yankaskas BC. Analysis of cancer missed at screening mammography. Radiology 1992; 184: 613-7.

4. Adriaenssens N, Belsack D, Buyl R, Ruggiero L, Breucq C, De Mey J, Lieven $P$, Lamote J. Ultrasound elastography as an objective diagnostic measurement tool for lymphoedema of the treated breast in breast cancer patients following breast conserving surgery and radiotherapy. Radiol Oncol 2012 46: 284-95

5. Zebic-Sinkovec M, Hertl K, Kadivec M, Cavlek M, Podobnik G, Snoj M Outcome of MRI-guided vacuum-assisted breast biopsy - initial experience at Institute of Oncology Ljubljana, Slovenia. Radiol Oncol 2012; 46: 97-105.

6. Agnese DM. Advances in breast imaging. Surg Technol Int 2005; 14: 51-6.

7. Scheidhauer K, Walter C, Seemann MD. FDG PET and other imaging modalities in the primary diagnosis of suspicious breast lesions. Eur J Nucl Med Mol Imaging 2004; 31: 70-9.

8. Thurlimann B, Muller A, Senn HJ. Management of primary breast cancer: an update. Onkologie 2004; 27: 175-9.

9. Rausch DR, Hendrick RE. How to optimize clinical breast MR imaging practices and techniques on your 1.5-T system. Radiographics 2006; 26: 1469-84.

10. Berg WA, Gutierrez L, NessAiver MS, Carter WB, Bhargavan M, Lewis RS, et al. Diagnostic accuracy of mammography, clinical examination, US, and MR imaging in preoperative assessment of breast cancer. Radiology 2004; 233: $830-49$.

11. Winnekendonk G, Krug B, Warm M, Gohring UJ, Mallmann P, Lackner K. Diagnostic value of preoperative contrast-enhanced MR imaging of the breast. Rofo 2004; 176: 688-93.

12. Antoch G, Vogt FM, Freudenberg LS, Nazaradeh F, Goehde SC, Barkhausen J, et al. Whole-body dual-modality PET/CTand whole-body MRI for tumor staging in oncology JAMA 2003; 290: 3199-206.

13. Czernin J, Allen-Auerbach M, Schelbert HR. Improvements in cancer staging with PET/CT: literature-based evidence as of September 2006. J Nucl Med 2007; 48 (Suppl 1): 78S-88S

14. Blodgett TM, Meltzer CC, Townsend DW. PET/CT: form and function. Radiology 2007; 242: 360-85.

15. Zangheri B, Messa C, Picchio M, Gianolli L, Landoni C, Fazio F. PET/CT and breast cancer. Eur J Nucl Med Mol Imaging 2004; 31 (Suppl 1): 135S-142S.

16. Tatsumi M, Cohade C, Mourtzikos KA, Fishman EK, Wahl RL. Initial experience with FDG-PET/CT in the evaluation of breast cancer. Eur I NuCl Med Mol Imaging 2006; 33: 254-62.
17. Hodolic M. Role of F-18-choline PET/CT in evaluation of patients with prostate carcinoma. Radiol Oncol 2011; 45: 17-21.

18. Kim JS, Jeong YJ, Sohn MH, Jeong HJ, Lim ST, Kim DW, et al. Usefulness of F-18 FDG PET/CT in subcutaneous panniculitis-like T cell lymphoma: disease extent and treatment response evaluation. Radiol Oncol 2012; 46: 279-83.

19. Radan L, Ben-Haim S, Bar-Shalom R, Guralnik L, Israel O. The role of FDG-PET/CT in suspected recurrence of breast cancer. Cancer 2006; 107: 2545-51.

20. Beresford M, Lyburn I, Sanghera B, Makris A, Wong WL. Serial integrated ${ }^{18} \mathrm{~F}$-fluorodeoxythymidine PET/CT monitoring neoadjuvant chemotherapeutic response in invasive ductal carcinoma. Breast J 2007; 13: 424-5.

21. Li D, Chen JH, Wang J, Ling R, Yao Q, Wang L. Value of fused F-18 FDG PET/ CT images in predicting efficacy of neoadjuvant chemotherapy on breast cancer. [in Chinese]. Ai Zheng 2007; 26: 900-4.

22. Reddy DH, Mendelson EB. Incorporating new imaging models in breast cancer management. Curr Treat Options Oncol 2005; 6: 135-45.

23. Fischer U, Kopka L, Grabbe E. Breast carcinoma: effect of preoperative contrastenhanced MR imaging on the therapeutic approach. Radiology 1999; 213: 881-8

24. Heusner TA, Freudenberg LS, Kuehl H, Hauth AM, Haibach PV, Forsting M, et al. Whole-body PET/CT-mammography for staging breast cancer: initial results. Br J Radiol 2008; 81: 743-8.

25. Ko BS, Noh WC, Kang SS, Park BW, Kang EY, Paik NS, et al. Changing patterns in the clinical characteristics of Korean breast cancer from 1996-2010 using an online nationwide breast cancer database. J Breast Cancer 2012; 15: $393-400$.

26. Andersson I. Mammographic screening and mortality from breast cancer mammographic screening trial. Br J Med 1998; 297: 943-8.

27. Lehman $C D$, Blume JD, Weatherall $P$, Thickman $D$, Hylton $N$, Warner $E$, et al. Screening women at high risk for breast cancer with mammography and magnetic resonance imaging. Cancer 2005; 103: 1898-905.

28. Friedrich M. MRI of the breast: state of the art. Eur Radiol 1998; 8: 707-25.

29. Bedrosian I, Mick R, Orel SG, Schnall MD, Reynolds C, Spitz F, et al. Changes in the surgical management of patients with breast carcinoma based on preoperative magnetic resonance imaging. Cancer 2003; 98: 468-73.

30. Orel SG, Schnall MD. MR imaging of the breast for detection, diagnosis and staging of breast cancer. Radiology 2001; 220: 3-30.

31. Schelfout K, Van Goethem M, Kersschot E, Colpaert C, Schelfhout AM Leyman $P$, et al. Contrast-enhanced MR imaging of breast lesions and effect on treatment. Eur J Surg Oncol 2004; 30: 501-7.

32. Hollingsworth AB, Stough RG, O'Dell CA, Brekke CE. Breast magnetic resonance imaging for preoperative locoregional staging. Am J Surg 2008; 196: 389-97.

33. Liberman L, Morris EA, Lee MJ, Kaplan JB, LaTrenta LR, Menell JH, et al. Breast lesions detected on MR imaging: features and positive predictive value. Am J Roentgenol 2002; 179: 171-8.

34. Heusner TA, Kuemmel S, Umutlu L, Koeninger A, Freudenberg LS, Hauth $\mathrm{AM}$, et al. Breast cancer staging in a single session: whole-body PET/CT mammography. J Nucl Med 2008; 49: 1215-22.

35. Samson DJ, Flamm CR, Pisano ED, Aronson N. Should FDG PET be used to decide whether a patient with an abnormal mammogram or breast finding at physical examination should undergo biopsy? Acad Radiol 2002; 9: 773-83. 